\title{
Correction to: Oral inoculation of ultraviolet-irradiated Eimeria species oocysts protects chickens against coccidiosis
}

\author{
Saeed A. El-Ashram ${ }^{1,2}$ - Shawky M. Aboelhadid ${ }^{3}$ - Sahar M. Gadelhaq ${ }^{4} \cdot$ Walid M. Arafa $^{3}$. \\ Abdel-Razik H. Abdel-Razik ${ }^{5}$. Salama A S Shany ${ }^{6} \cdot$ Khaled T. Abdelaziz ${ }^{7,8}$
}

Published online: 3 January 2020

(C) Springer-Verlag GmbH Germany, part of Springer Nature 2020

\section{Correction to: Parasitology Research 118(11): 3173-3183} https://doi.org/10.1007/s00436-019-06455-y

The author name Salama Abohamra in the original published version of this article should have been Salama Abohamra Sayed Shany.

This article is part of the Topical Collection on Erratum

The online version of the original article can be found at https://doi.org/ 10.1007/s00436-019-06455-y

Saeed A. El-Ashram

saeed_elashram@yahoo.com

$\triangle$ Shawky M. Aboelhadid

Drshawky2001@yahoo.com

1 College of Life Science and Engineering, Foshan University, 18 Jiangwan Street, Foshan 528231, Guangdong Province, China

2 Faculty of Science, Kafrelsheikh University, Kafr el-Sheikh 33516, Egypt

3 Department of Parasitology, Faculty of Veterinary Medicine, Beni-Suef University, Beni-Suef 62511, Egypt

4 Department of Parasitology, Faculty of Veterinary Medicine, Minia University, El-Minia, Egypt

5 Department of Histology, Faculty of Veterinary Medicine, Beni-Suef University, Beni-Suef 62511, Egypt

6 Department of Poultry Diseases, Faculty of Veterinary Medicine, Beni-Suef University, Beni-Suef 62511, Egypt

7 Department of pathology, Ontario Veterinary College, University of Guelph, Ontario N1G 2W1, Canada

8 Department of pathology, Faculty of Veterinary Medicine, Beni-Suef University, Beni-Suef 62511, Egypt 